\title{
Assessing the trophic state of Linhos lake: a first step towards ecological rehabilitation
}

\author{
R. Pereira*†, A. M. V. M. Soares ${ }^{\dagger \ddagger}$, R. Ribeiro ${ }^{\ddagger}$ and F. Gonçalves ${ }^{\dagger}$ \\ ${ }^{\dagger}$ Departamento de Biologia, Universidade de Aveiro, Campus Universitario de Santiago, 3810-193 \\ Aveiro, Portugal \\ ¥Instituto do Ambiente e Vida, Departamento de Zoologia, Universidade de Coimbra, Portugal
}

Received 1 June 2000; accepted 8 October 2001

\begin{abstract}
Lack of recognition of the value of wetlands has led to the loss of considerable areas of these ecosystems in the past. Linhos lake (Figueira da Foz, Portugal) is a good example of one of these ecosystems, in which human intervention was responsible for its environmental degradation and led to its precocious terrestrialization. Physico-chemical conditions and zooplankton community structure were studied in Linhos lake, in order to evaluate ecosystem functioning and to acquire baseline information. The system is characterised by high oxygen depletion. Spatial heterogeneity was confirmed by the existence of significant differences in total densities for the three zooplanktonic groups. Rotifers were the most abundant group attaining their maximum density in April (2251.1 ind/l). Keratella quadrata, K. cochlearis, Polyarthra vulgaris, Filinia terminalis and Hexarthra mira were the main abundant species. Correspondence analysis suggested temperature as the main controlling factor in species seasonality. In order to prevent the precocious disappearance of the lake some restoration measures were proposed based on zooplankton community structure.
\end{abstract}

(C) 2002 Elsevier Science Ltd.

Keywords: zooplankton community structure, lake terrestrialization, ecological rehabilitation adaptative management.

\section{Introduction}

At the beginning of the last century numerous small Portuguese freshwater lakes, between Oporto and Lisbon, near the coast, were drained in an attempt to eradicate the malaria vector that enjoyed, in central Portugal, ideal breeding habitats. One of these lakes, Linhos lake, almost disappeared in the process. More recently, the lake has been affected by the removal of groundwater by a paper mill. These activities contributed to a significant reduction of its surface area and depth and led to the development of a dense macrophyte community. Additionally, the enrichment of the sediments with organic matter provided annually by senescent macrophytes and their subsequent decomposition, depleted the oxygen and enhanced

\footnotetext{
* Corresponding author. Email: ruthp@bio.ua.pt
}

nutrient loading, particularly phosphorus, within the overlying water which thus became eutrophic (Pokorný, 1994). The loss of submerged vegetation and reduction in biodiversity (Michaud et al., 1979; Hosper and Jagtman, 1990; Brönmark and Weisner, 1992; Wetzel, 1993; Klinge et al., 1995), the occurrence of algal blooms (Michaud et al., 1979; Brix and Schierup, 1989; Hosper and Jagtman, 1990; Klinge et al., 1995), and increase in turbidity (Klinge et al., 1995) are some of the characteristics associated with eutrophic shallow lakes, which seriously compromise their wildlife and recreational use. The eutrophication process remains a major problem in Linhos, as in most of the freshwater lakes of Portugal, and improvement strategies are required (e.g. Vasconcelos, 1994; Silva et al., 1997; Gonçalves et al., 1996).

The understanding of ecosystem functioning is essential to decide which restoration measures are appropriate (Wetzel, 1993). However, planning and 
restoration of a natural area need not be inhibited by a lack of scientific information, if an adaptative management process is adopted. This is a process in which resource managers plan management activities continually, monitor and adjust them to meet their goals (Marcin, 1995).

Adequate restoration measures for many lakes should focus on the control of emergent macrophyte standing crops. Wetlands dominated by perennial macrophytes such as Phragmites, are one of the world's most productive ecosystems (Björk, 1994a). Its overgrowth is continually stimulated by the recycling of nutrients from the sediments, which constrains the number of trophic levels and topdown forces that in turn regulate their standing crops (Power, 1992; Björk, 1994a). Commonly, additional measures should also be taken to reduce phosphorus internal loading. Phosphorus is a key element in freshwater lakes (Hosper and Jagtman, 1990; Seip, 1994) since its concentration in those systems is lower than its biological demand (Wetzel, 1993). Zooplankton constitutes a sensitive tool for monitoring eutrophication (Pejler, 1983; Magadza, 1994; Silva et al., 1997), because populations react immediately to changes in trophic status. The information obtained from this study will allow the monitoring of the effects of intended restoration measures.

Eutrophication usually affects both the physical and chemical environment and can lead to significant changes in the phytoplankton and zooplankton communities' structure (Flores and Barone, 1994; Uku and Mavuti, 1994). Generally, this process results in a zooplankton community dominated by rotifers and small bodied cladocerans (Gliwicz, 1990; Matsubara, 1993; Ejsmont-Karabin, 1995) which are less vulnerable to feeding interference by large inedible algae species (Gliwicz, 1990; Auer et al., 1990) and capable of feeding on detritus and bacterial size particles (Bogdan and Gilbert, 1982). The aim of the present study was to develop the first set of measures for the ecological rehabilitation of Linhos lake. In order to meet this goal, the zooplankton community structure was characterized and inferences made about how the physical and chemical conditions of the lake affect it.

\section{Material and methods}

\section{Study site}

The Lake is a small ( $10 \mathrm{ha}$ ), shallow (1.43 m mean depth; $2.3 \mathrm{~m}$ maximum depth) and eutrophic water

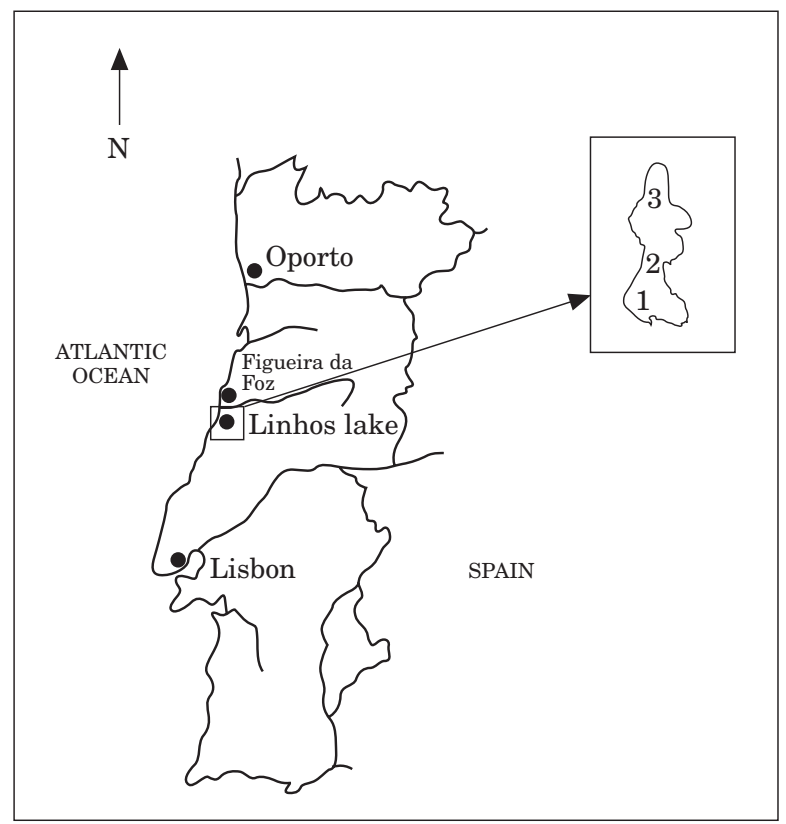

Figure 1. Location of Linhos lake.

body, located $15 \mathrm{~km}$ South of Figueira da Foz (Portugal) (Figure 1). A large community of emergent macrophytes was present, with Phragmites australis (CAV.) Trin. Ex. Steudel and Typha latifolia L. as the dominant species. At the end of the growing season, senescent macrophytes fall into the lake forming a completely anoxic organic layer (approximately $1.5 \mathrm{~m}$ thickness, personal observation). The brownish coloured water of the Lake is indicative of the presence of high concentrations of dissolved organic compounds. Submerged macrophytes were absent. A dense forest consisting largely of Pinus pinaster Aiton and Eucalyptus globulus Labill and a marsh zone, characterised by high species diversity, surround the lake, isolating it from human activity, hence offering good conditions for wildlife, especially migratory birds.

\section{Sampling procedures}

Three sampling sites were defined in the Lake. Sites 1 and 3 were located near the emergent vegetation and site 2 in open water. Samples for water chemistry and zooplankton determinations were collected monthly from the surface, on each sampling site, during 12 months. Dissolved oxygen, Secchi disk transparency, conductivity, temperature and $\mathrm{pH}$ were recorded in situ every month. Temperature $\left( \pm 0.5^{\circ} \mathrm{C}\right)$ and $\mathrm{pH}( \pm 0.02 \mathrm{pH})$ were measured by Jenway $3150 \mathrm{pH}$ meter. The conductivity was measured by a WTW LF92 conductivity 
meter. Dissolved oxygen $(\mathrm{mg} / \mathrm{l}, \pm 0 \cdot 1 \mathrm{mg} / \mathrm{l})$ was measured by a WTW OXI92 oxygen meter. From March to December 1996 vertical profiles for dissolved oxygen were obtained measuring this parameter at $25 \mathrm{~cm}$ intervals. Ammonium $(\mathrm{mg} / \mathrm{l})$ was determined by the Nessler method, nitrate $(\mathrm{mg} / \mathrm{l})$ by cadmium reduction, nitrite $(\mathrm{mg} / \mathrm{l})$ by a colorimetric method and phosphorus $(\mathrm{mg} / \mathrm{l})$ following the ascorbic acid method (APHA et al., 1989). For chlorophyll- $a$ analysis, water samples were filtered through Whatman GF/C filters $(0.45 \mu \mathrm{m}$ porosity; $47 \mathrm{~mm}$ diameter). Filters were ground in $90 \%$ acetone and stored at $4^{\circ} \mathrm{C}$ for $24 \mathrm{~h}$. After centrifugation, absorbency was measured at $664 \mathrm{~nm}$ and $750 \mathrm{~nm}$ before adding $\mathrm{HCl}$ and at $750 \mathrm{~nm}$ and $665 \mathrm{~nm}$ after acidification by a Jenway 6100 spectrophotometer. Chlorophyll- $a$ concentration was calculated and total suspended solids determined according to the methods of the APHA, AWWA and WPCF (1989).

Zooplankton samples were collected in a $1 \mathrm{~L}$ bottle. Ten liters of water were filtered, per sample, through a plankton net ( $20 \mu \mathrm{m}$ mesh). Each sample was immediately preserved in borate buffered with $4 \%$ formalin. Three replicates per sampling site were collected for rotifer observation, and six for cladocerans and copepods.

In the laboratory, copepods (Dussart, 1969) and cladocerans (Amoros, 1984; Scourfield and Harding, 1966) were identified and counted under a dissection microscope $(50 \times)$. For rotifers, sedimentation chambers were used, under an inverted microscope (Ruttner-Kolisko, 1974).

\section{Statistical analysis}

One-way ANOVA was used to detect the existence of significant differences among sites for environmental parameters and in total densities of rotifers, cladocerans and copepods, during each month. In order to meet the basic assumptions required for a valid analysis of variance (Zar, 1996), total densities of the three zooplankton groups were transformed by the equation: $x^{\prime}=\log (x+1)$.

The zooplankton assemblages were examined for their taxonomic diversity using the Shannon-Wiener index $\left(\mathrm{H}^{\prime}\right)$. An equitability index $\left(\mathrm{J}^{\prime}\right)$ was also calculated to complement the information provided by the diversity index, since the $\mathrm{J}^{\prime}$ is useful to detect the presence of new species in the system (Legendre and Legendre, 1979; Washington, 1984). These indices were applied to genera since in some cases identification to species level was not possible (Washington, 1984).
Resemblances between the zooplankton assemblages were analysed by correspondence analysis (CA) using NT-SYS 1.8 (Rohlf, 1992). Months and species were plotted together to ascertain out the existence of gradients leading to species distribution.

To assess positive links between the most abundant taxa and environmental parameters, simple regression analysis was performed with the transformed data. Regression models were compared (regression coefficients and intercepts) by analysis of covariance. Whenever null hypothesis was rejected, a multiple comparison test (Tukey test) was used to determine which slopes and intercepts differed from each other (Zar, 1996).

\section{Results}

\section{Environmental parameters}

Linhos lake (henceforth the Lake) is characterised by strong dissolved oxygen depletion. Dissolved oxygen concentrations vary between $0.3 \mathrm{mg} / \mathrm{l}$ and $5.3 \mathrm{mg} / \mathrm{l}$ (Figure 2a). The highest values were recorded in March $(5.3 \mathrm{mg} / \mathrm{l}$, sites 1 and 3 ) and October ( $5.3 \mathrm{mg} / \mathrm{l}$, site 3 ). Dissolved oxygen profiles declined with increasing depth, indicative of the widespread anoxia in the deep water and surface sediments (Table 1 ).

Water temperature ranged between $7.3^{\circ} \mathrm{C}$ (December) and $21.4^{\circ} \mathrm{C}$ (July) (Table 2). The Lake did not exhibit temperature stratification during the study period (Table 2).

The $\mathrm{pH}$ values ranged between 5.9 and 7.9. The higher values were registered in July (7.93) and October (7.65) (Table 2).

Specific conductance in lake water averaged $\leq 870 \mu \mathrm{S} / \mathrm{cm}$ (Table 2); a gradual increase from the beginning to the end of the study was observed.

Chlorophyll- $a$ concentrations peaked in January (11.0 $\mu / 1$, site 1$)$. High values were also registered in March (9.6 $\mu / 1$, site 2; $7.6 \mu \mathrm{g} / \mathrm{l}$, site 3), June (7.4 $\mu \mathrm{g} / \mathrm{l}$, site $1 ; 8.0 \mu \mathrm{g} / \mathrm{l}$, site 2$)$, and November $(10.4 \mu \mathrm{g} / \mathrm{l}$, site $1 ; 10.0 \mu \mathrm{g} / \mathrm{l}$, site 3 ) (Figure 2b). Seasonal variation in chlorophyll was not observed.

Nitrites were the least abundant nitrogen compound. Their concentrations were higher in the first four months, especially in February $(15 \mu \mathrm{g} / \mathrm{l}$; site 1 ). Values of $0 \mu \mathrm{g} / \mathrm{l}$ were obtained during late spring and summer (Figure 2c).

As with nitrites, nitrates showed highest concentrations during winter, decreasing gradually in spring, reaching lowest values in June $(0.2 \mathrm{mg} / \mathrm{l}$, site 2). During the final six months 

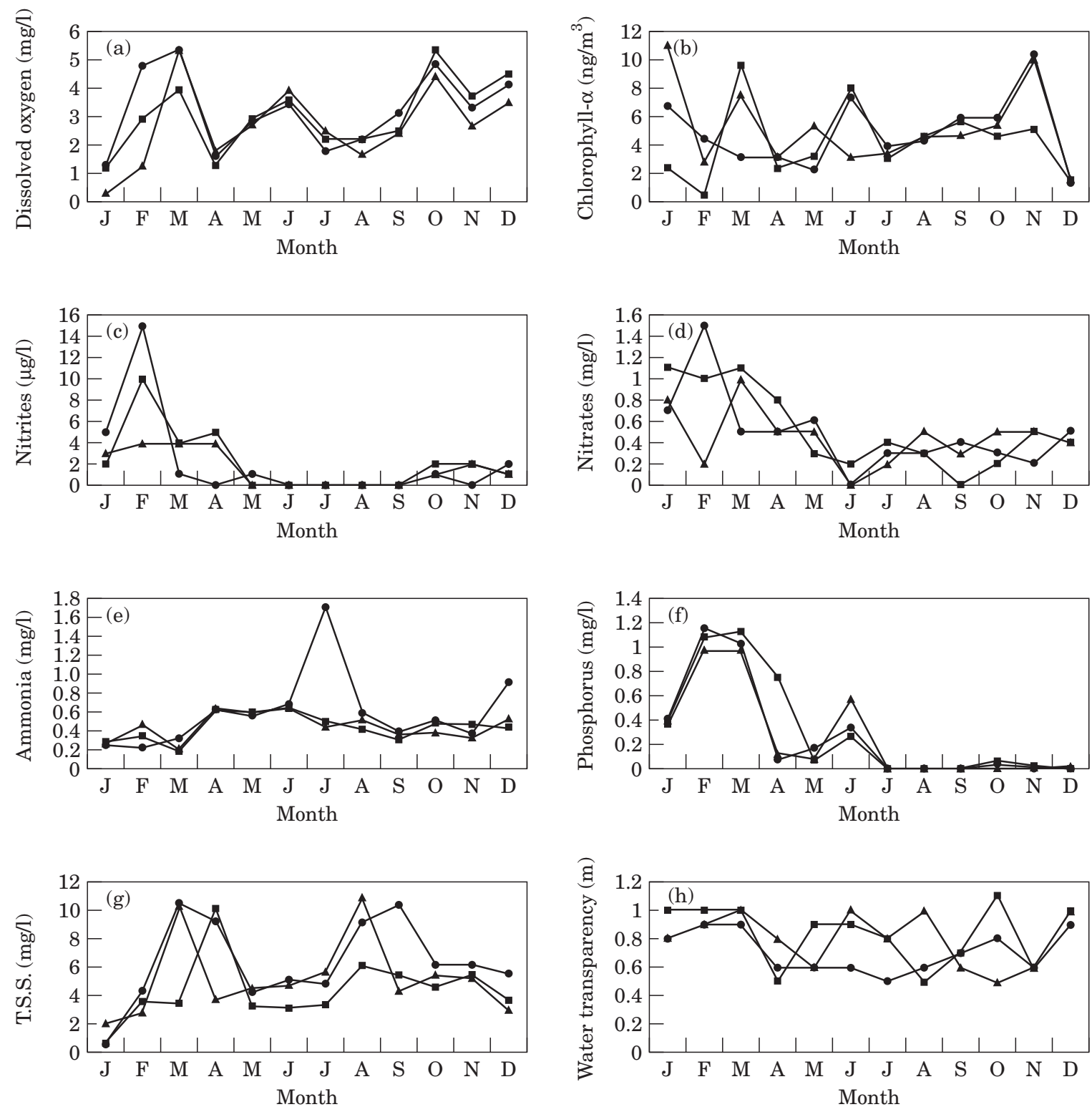

Figure 2. Annual variation, within each sampling site of: (a) dissolved oxygen; (b) chlorophyll-a; (c) nitrite concentrations; (d) nitrate concentrations; (e) ammonia concentrations; (f) phosphorus concentrations; (g) total suspended solids

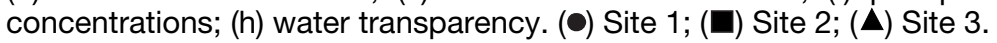

(July-December), nitrates displayed almost no variation (Figure 2d).

Ammonia concentrations showed small variations throughout the year, with the exception of two peak values: July (1.7 mg/l, site 1) and December $(0.91 \mathrm{mg} / \mathrm{l}$, site 1$)$ (Figure $2 \mathrm{e})$.

Phosphorus concentrations were higher in February $(\bar{x}=1.07 \mathrm{mg} / \mathrm{l})$ and March $(\overline{\mathrm{x}}=1.04 \mathrm{mg} / \mathrm{l})$. Throughout the last six months of the year, concentrations were very low, varying between $0.06 \mathrm{mg} / \mathrm{l}$ and $0.001 \mathrm{mg} / \mathrm{l}$ (Figure 2f); suggesting possible removal from the water through biological uptake by macrophytes and phytoplankton. The last group was characterised in those months by a great abundance of large edible algae (Pereira, personal observation). Bacteria and microplankton could also be responsible, through rapid immobilization of phosphorus from surface waters, during the growing season (Freedman, 1989).

Four peaks of Total Suspended Solids (TSS) were recorded in March $(10.4 \mathrm{mg} / \mathrm{l}$, site $1 ; 10.3 \mathrm{mg} / \mathrm{l}$, site 3$)$, April $(9.2 \mathrm{mg} / \mathrm{l}$, site $1 ; 10.1 \mathrm{mg} / \mathrm{l}$ site 2 ), August $(9.1 \mathrm{mg} / \mathrm{l}$, site $1 ; 10.9 \mathrm{mg} / \mathrm{l}$, site 3$)$ and 
Table 1. Dissolved oxygen concentration ( $\mathrm{mg} / \mathrm{l})$ measured, at $25 \mathrm{~cm}$ intervals, at each sampling site

\begin{tabular}{|c|c|c|c|c|c|c|c|c|c|c|}
\hline & Mar & Apr & May & Jun & Jul & Aug & Sep & Oct & Nov & Dec \\
\hline Depth (cm) & \multicolumn{10}{|c|}{ Site 1} \\
\hline 25 & $5 \cdot 3$ & 1.6 & $2 \cdot 8$ & 3.4 & $1 \cdot 8$ & $2 \cdot 2$ & $3 \cdot 1$ & 4.8 & 3.3 & $4 \cdot 1$ \\
\hline 50 & 4.6 & 1.4 & $2 \cdot 3$ & 3.0 & $1 \cdot 7$ & $2 \cdot 1$ & $3 \cdot 1$ & $3 \cdot 3$ & $3 \cdot 2$ & $4 \cdot 1$ \\
\hline 75 & 4.4 & 1.4 & 0.9 & $1 \cdot 2$ & $1 \cdot 7$ & $1 \cdot 7$ & 2.9 & 1.3 & $3 \cdot 1$ & 3.9 \\
\hline \multirow[t]{2}{*}{100} & $2 \cdot 0$ & $1 \cdot 2$ & $0 \cdot 1$ & 0.3 & 0.3 & 0.8 & 0.7 & 0.1 & 0.2 & $3 \cdot 3$ \\
\hline & \multicolumn{10}{|c|}{ Site 2} \\
\hline 25 & 3.9 & 1.4 & 2.9 & 3.6 & $2 \cdot 2$ & $2 \cdot 2$ & 2.5 & $5 \cdot 3$ & 3.7 & 4.5 \\
\hline 50 & $2 \cdot 8$ & 1.4 & $2 \cdot 7$ & $3 \cdot 1$ & $2 \cdot 0$ & $2 \cdot 1$ & 2.5 & $3 \cdot 7$ & 3.6 & 4.5 \\
\hline 75 & 1.9 & 1.3 & 0.8 & 1.3 & 1.6 & 1.8 & 2.6 & 1.8 & 3.6 & 4.4 \\
\hline \multirow[t]{2}{*}{100} & 0.8 & $1 \cdot 2$ & 0.1 & 0.5 & 0.3 & 0.4 & 1.4 & 0.5 & $3 \cdot 2$ & 4.4 \\
\hline & \multicolumn{10}{|c|}{ Site 3} \\
\hline 25 & $5 \cdot 3$ & 1.8 & $2 \cdot 7$ & 3.9 & 2.5 & 1.8 & 2.4 & 4.4 & $2 \cdot 7$ & 3.5 \\
\hline 50 & 4.0 & 1.5 & $2 \cdot 4$ & 3.7 & $2 \cdot 1$ & $1 \cdot 8$ & $2 \cdot 3$ & $2 \cdot 6$ & $2 \cdot 7$ & 3.5 \\
\hline 75 & 1.6 & $1 \cdot 1$ & 1.0 & $2 \cdot 6$ & $1 \cdot 8$ & 1.7 & $2 \cdot 3$ & 0.7 & 2.7 & 3.3 \\
\hline 100 & 0.4 & 1.3 & 0.1 & 0.2 & 0.2 & 1.2 & 2 & 0.1 & 2.7 & $2 \cdot 8$ \\
\hline
\end{tabular}

Table 2. Monthly variation of water temperature $\left({ }^{\circ} \mathrm{C}\right), \mathrm{pH}$ and Conductivity $(\mu \mathrm{S} / \mathrm{cm})$ at each sampling site

\begin{tabular}{|c|c|c|c|c|c|c|c|c|c|}
\hline & \multicolumn{3}{|c|}{ Site 1} & \multicolumn{3}{|c|}{ Site 2} & \multicolumn{3}{|c|}{ Site 3} \\
\hline & $\begin{array}{c}\text { Temp. } \\
\left({ }^{\circ} \mathrm{C}\right)\end{array}$ & $\mathrm{pH}$ & $\begin{array}{l}\text { Cond. } \\
(\mu \mathrm{S} / \mathrm{cm})\end{array}$ & $\begin{array}{c}\text { Temp. } \\
\left({ }^{\circ} \mathrm{C}\right)\end{array}$ & $\mathrm{pH}$ & $\begin{array}{l}\text { Cond. } \\
(\mu \mathrm{S} / \mathrm{cm})\end{array}$ & $\begin{array}{c}\text { Temp } \\
\left({ }^{\circ} \mathrm{C}\right)\end{array}$ & $\mathrm{pH}$ & $\begin{array}{c}\text { Cond. } \\
(\mu \mathrm{S} / \mathrm{cm})\end{array}$ \\
\hline Jan & 9.9 & 6.2 & 504 & 11.2 & $6 \cdot 3$ & 508 & $10 \cdot 3$ & $6 \cdot 1$ & 518 \\
\hline Feb & 10.9 & $6 \cdot 0$ & 529 & 10.6 & 6.4 & 533 & $10 \cdot 6$ & $6 \cdot 4$ & 535 \\
\hline Mar & $17 \cdot 3$ & $6 \cdot 0$ & 498 & $18 \cdot 7$ & $6 \cdot 3$ & 537 & 17 & 6.4 & 539 \\
\hline Apr & $13 \cdot 8$ & 7.0 & 535 & 14.4 & 7.0 & 555 & 14.8 & $6 \cdot 8$ & 547 \\
\hline May & $17 \cdot 8$ & 6.3 & 506 & $18 \cdot 2$ & $6 \cdot 1$ & 528 & 18.6 & $6 \cdot 1$ & 514 \\
\hline Jun & $19 \cdot 6$ & 6.7 & 543 & 20.1 & 6.5 & 557 & 20 & 6.4 & 556 \\
\hline Jul & 21.1 & 7.9 & 569 & 21.4 & 7.4 & 573 & 21.4 & $7 \cdot 6$ & 572 \\
\hline Aug & 21.1 & 6.9 & 589 & $21 \cdot 2$ & 7.0 & 589 & $21 \cdot 2$ & 6.8 & 591 \\
\hline Sep & 17 & 6.9 & 635 & 16.9 & 6.9 & 643 & 16.9 & 6.9 & 642 \\
\hline Oct & $17 \cdot 2$ & 7.7 & 643 & $17 \cdot 2$ & $7 \cdot 1$ & 648 & $17 \cdot 3$ & $7 \cdot 1$ & 640 \\
\hline Nov & 14.2 & 5.9 & 849 & $13 \cdot 3$ & $6 \cdot 2$ & 851 & $13 \cdot 8$ & $6 \cdot 0$ & 870 \\
\hline Dec & $7 \cdot 3$ & $6 \cdot 1$ & 852 & 7.9 & $6 \cdot 1$ & 849 & $7 \cdot 8$ & $6 \cdot 1$ & 840 \\
\hline
\end{tabular}

September (10.4 mg/l, site 1) (Figure $2 \mathrm{~g}$ ). These values could be attributed to the high density of both Keratella quadrata, and of the large edible phytoplankton species Ceratium sp. (Pereira, personal observation), during spring and summer, respectively.

The highest values of water transparency were registered in January ( $1 \mathrm{~m}$, site 2$)$, March ( $1 \mathrm{~m}$, site 2 and 3 ), June ( $1 \mathrm{~m}$, site 3 ), August ( $1 \mathrm{~m}$, site 3 ), October ( $1.1 \mathrm{~m}$, site 2$)$ and December $(1 \mathrm{~m}$, site 2 and 3) (Figure 2h). Surprisingly, even during periods of low chlorophyll- $a$ concentrations (April, May and July) transparency was low, which seems to be indicative of the possibility of water transparency being governed by other factors, such as dissolved organic compounds, zooplankton or the resuspension of sediment particles (Hosper and Jagtman, 1990; Wetzel, 1993).

\section{Zooplankton communities}

Rotifers were the most abundant group, representing $68.9-99.9 \%$ of the total zooplankton, followed by copepods $(<20.8 \%)$ and cladocerans $(<10.4 \%)$. Eighteen species and 21 genera of rotifers were identified; K. quadrata, K. cochlearis, Filinia terminalis, Hexarthra mira were the most abundant taxa (Table 3). Anuraeopsis fissa, Ascomorpha ecaudis and Synchaeta spp. also showed consistently high densities. Rotifers presented three density peaks in April, August and November (Figure 3a). The April peak (site 1) was mainly due to K. quadrata (99\% of total density). The second peak (site 1) was due to an increase in the density of three species: Filinia terminalis (54.08\%), $K$. cochlearis $(28.6 \%)$ and H. mira (10.1\%). K. cochlearis was also responsible for the third peak (site 1), representing $87.7 \%$ of 
Table 3. Rotifers, cladocerans and copepods identified for Linhos lake

\begin{tabular}{|c|c|c|c|c|c|c|c|c|c|c|c|c|}
\hline & $\mathrm{J}$ & $\mathrm{F}$ & $\mathrm{M}$ & A & $\mathrm{M}$ & $\mathrm{J}$ & $J$ & A & $\mathrm{S}$ & $\mathrm{O}$ & $\mathrm{N}$ & D \\
\hline \multicolumn{13}{|l|}{ Rotatoria } \\
\hline Brachionus patulus Muller & & & & & & $\circ$ & $\circ$ & $\circ$ & $\circ$ & & & \\
\hline Brachionus angularis Gosse & & $\circ$ & $\circ$ & & & & & & & & & \\
\hline Brachionus falcatus Zacharias & & $\circ$ & & & & & & & & & & \\
\hline Brachionus bidentatus Andersen & & $\circ$ & & & & & & & & & & \\
\hline Brachionus calyciflorus (Pallas) & & & & & & & o & $\circ$ & $\circ$ & $\circ$ & & \\
\hline Platyas quadricornis Ehrenberg & $\circ$ & & $\circ$ & $\circ$ & & & $\circ$ & $\circ$ & $\circ$ & $\circ$ & $\circ$ & \\
\hline Keratella quadrata Muller & $\circ$ & $\circ$ & + & ++ & $\circ$ & $\circ$ & $\circ$ & $\circ$ & $\circ$ & $\circ$ & $\circ$ & $\circ$ \\
\hline Keratella valga Carlin & & & $\circ$ & & $\circ$ & & & & & & & \\
\hline Keratella cochlearis Gosse & $\circ$ & $\circ$ & $\circ$ & $\circ$ & $\circ$ & + & + & ++ & + & + & ++ & ++ \\
\hline Anuraeopsis fissa Gosse & & $\circ$ & $\circ$ & + & $\circ$ & $\circ$ & $\circ$ & $\circ$ & $\circ$ & $\circ$ & & $\circ$ \\
\hline Euchlanis spp. & $\circ$ & $\circ$ & $\circ$ & $\circ$ & $\circ$ & $\circ$ & $\circ$ & $\circ$ & $\circ$ & $\circ$ & $\circ$ & $\circ$ \\
\hline Lepadella spp. & $\circ$ & $\circ$ & $\circ$ & $\circ$ & $\circ$ & $\circ$ & + & $\circ$ & $\circ$ & $\circ$ & $\circ$ & $\circ$ \\
\hline Squatinella sp. & $\circ$ & $\circ$ & $\circ$ & $\circ$ & & $\circ$ & $\circ$ & $\circ$ & $\circ$ & & $\circ$ & $\circ$ \\
\hline Lecane quadridentata Ehrenberg & & & & & & $\circ$ & $\circ$ & $\circ$ & $\circ$ & $\circ$ & $\circ$ & \\
\hline Lecane spp. & $\circ$ & $\circ$ & $\circ$ & $\circ$ & $\circ$ & $\circ$ & $\circ$ & $\circ$ & $\circ$ & $\circ$ & $\circ$ & ० \\
\hline Mytilina sp. & $\circ$ & $\circ$ & $\circ$ & $\circ$ & $\circ$ & $\circ$ & $\circ$ & $\circ$ & $\circ$ & & $\circ$ & \\
\hline Trichotria sp. & & $\circ$ & $\circ$ & $\circ$ & $\circ$ & $\circ$ & $\circ$ & $\circ$ & $\circ$ & $\circ$ & $\circ$ & ० \\
\hline Monommata sp. & $\circ$ & & $\circ$ & $\circ$ & & $\circ$ & $\circ$ & $\circ$ & & $\circ$ & $\circ$ & \\
\hline Scaridium sp. & & $\circ$ & & $\circ$ & & $\circ$ & $\circ$ & $\circ$ & $\circ$ & & $\circ$ & \\
\hline Trichocerca elongata Gosse & & $\circ$ & $\circ$ & & & $\circ$ & $\circ$ & & & & $\circ$ & ० \\
\hline Trichocerca spp. & $\circ$ & $\circ$ & $\circ$ & $\circ$ & $\circ$ & ○ & $\circ$ & $\circ$ & $\circ$ & $\circ$ & $\circ$ & \\
\hline Ascomorpha ecaudis Perty & & $\circ$ & $\circ$ & ० & + & $\circ$ & ० & $\circ$ & $\circ$ & $\circ$ & $\circ$ & $\circ$ \\
\hline Asplancna sp. & & $\circ$ & & & & $\circ$ & ○ & $\circ$ & $\circ$ & $\circ$ & $\circ$ & \\
\hline Synchaeta spp. & & $\circ$ & $\circ$ & $\circ$ & + & + & + & + & + & + & + & ○ \\
\hline Polyarthra vulgaris Carlin & $\circ$ & $\circ$ & $\circ$ & $\circ$ & $\circ$ & + & $\circ$ & + & + & $\circ$ & $\circ$ & $\circ$ \\
\hline Testudinella sp. & $\circ$ & $\circ$ & $\circ$ & $\circ$ & $\circ$ & 0 & $\circ$ & & $\circ$ & & $\circ$ & ० \\
\hline Pompholix sp. & & $\circ$ & & & & & & & & & & \\
\hline Filinia hofmanni Koste & $\circ$ & & $\circ$ & $\circ$ & & & & & & & & \\
\hline Filinia terminalis Plate & & $\circ$ & $\circ$ & $\circ$ & & + & ++ & ++ & + & $\circ$ & $\circ$ & $\circ$ \\
\hline Filinia opoliensis Zacharias & $\circ$ & & $\circ$ & $\circ$ & $\circ$ & & & & & & & \\
\hline Hexarthra mira Hudson & $\circ$ & & & $\circ$ & $\circ$ & $\circ$ & $\circ$ & + & $\circ$ & $\circ$ & & \\
\hline \multicolumn{13}{|l|}{ Cladocera } \\
\hline Diaphanosoma brachyurum Liéven & & & & & $\circ$ & & & & & & & \\
\hline Ceriodaphnia pulchella Sars & $\circ$ & $\circ$ & $\circ$ & & $\circ$ & & & & & & & \\
\hline Bosmina longirostris (O. F. Muller) & $\circ$ & $\circ$ & $\circ$ & $\circ$ & $\circ$ & $\circ$ & $\circ$ & $\circ$ & $\circ$ & $\circ$ & $\circ$ & $\circ$ \\
\hline Alona protzi Hartwing & & $\circ$ & & & $\circ$ & $\circ$ & & & & & & \\
\hline Alona rectangula Sars & & $\circ$ & $\circ$ & $\circ$ & $\circ$ & $\circ$ & ○ & $\circ$ & $\circ$ & $\circ$ & $\circ$ & $\circ$ \\
\hline Alona tenuicaudis Sars & & & $\circ$ & $\circ$ & $\circ$ & 0 & & & & & & \\
\hline Alona weltneri Keilhack & & $\circ$ & & & $\circ$ & & & & & & & \\
\hline Alona costata Sars & $\circ$ & & $\circ$ & $\circ$ & $\circ$ & & $\circ$ & $\circ$ & $\circ$ & $\circ$ & $\circ$ & $\circ$ \\
\hline Alonella nana (Baird) & & & & $\circ$ & & & & $\circ$ & & $\circ$ & & $\circ$ \\
\hline Alonella exigua Lilljeborg & & $\circ$ & & & $\circ$ & & & & & & & \\
\hline Chydorus sphaericus Muller & & & $\circ$ & $\circ$ & & $\circ$ & & $\circ$ & & $\circ$ & & ० \\
\hline Ilyocryptus sordidus (Liéven) & & & & & & & & o & $\circ$ & $\circ$ & $\circ$ & \\
\hline \multicolumn{13}{|l|}{ Copepoda } \\
\hline Acanthocyclops robustus G.O. Sars & $\circ$ & $\circ$ & & $\circ$ & & ○ & $\circ$ & $\circ$ & & $\circ$ & & \\
\hline Copidodiaptomus numidicus Gurney & $\circ$ & $\circ$ & $\circ$ & $\circ$ & $\circ$ & $\circ$ & $\circ$ & $\circ$ & & & $\circ$ & \\
\hline Copepodits & $\circ$ & $\circ$ & $\circ$ & $\circ$ & $\circ$ & $\circ$ & o & $\circ$ & $\circ$ & $\circ$ & $\circ$ & $\circ$ \\
\hline Nauplii & $\circ$ & $\circ$ & $\circ$ & + & + & + & + & + & + & + & $\circ$ & + \\
\hline Harpaticoids & & $\circ$ & & $\circ$ & $\circ$ & $\circ$ & & & & $\circ$ & & $\circ$ \\
\hline
\end{tabular}

$(\circ-$ ind $/ /<10 ;+-10<$ ind $/ /<100 ;++-$ ind $/ \mathrm{l}>100)$.

total density (Figure 3a). Significant differences in density between sites were found in April $(P=0.0002 ; \mathrm{df}=2 ; F=48.1)$, August $(P=0.0008$; $\mathrm{df}=2 ; F=29 \cdot 2)$, and November $(P=0.0008 ; \mathrm{df}=2$; $F=29 \cdot 3$ ).
Copepods were represented by two species: Acanthocyclops robustus and Copidodiaptomus numidicus (Table 3). Maximum densities were recorded in April (42.6 ind/l, site 1), August (70.4 ind/l, site 1 ), and September ( $83.5 \mathrm{ind} / \mathrm{l}$, site 1$)$. These 

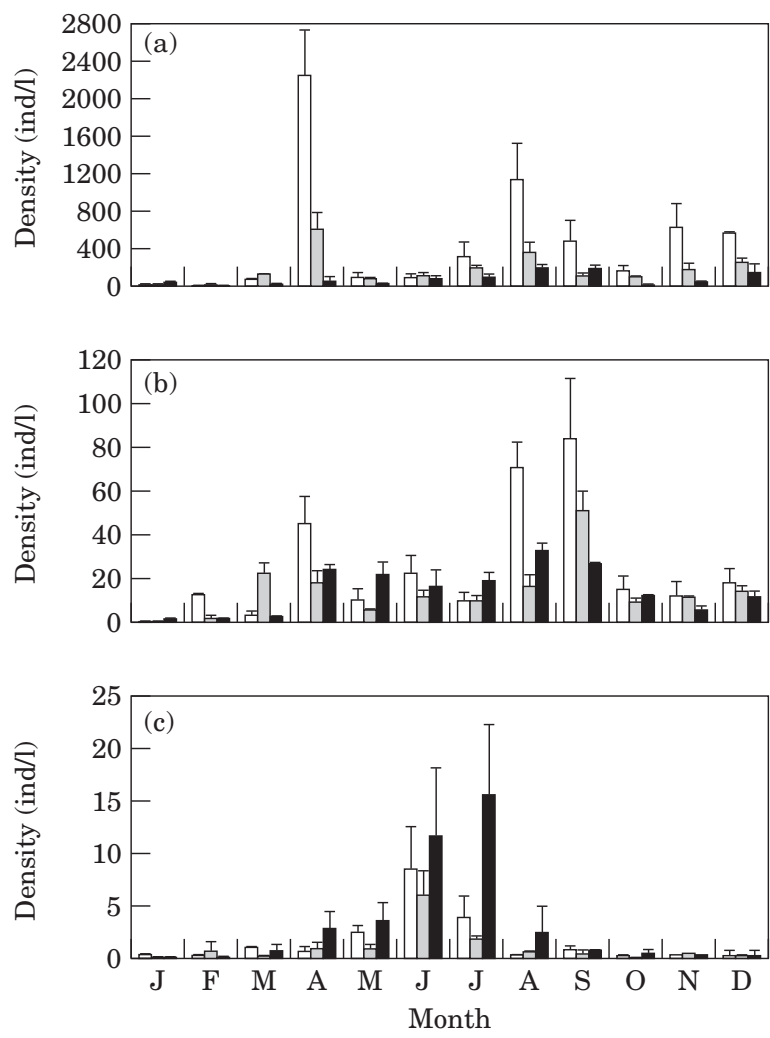

Figure 3. Annual variation, within each sampling site of densities of: (a) rotifers, (b) copepods and (c) cladocerans. $(\square)$ Site 1; $(\square)$ Site 2; $(\square)$ Site 3.

values can be mainly attributed to naupliar forms which represent $85.7 \%, 97.1 \%$ and $98.4 \%$ of total density, respectively (Figure 3b). Significant differences existed between sites in April $(P=0 ; \mathrm{df}=2 ; F=37.5)$, August $(P<0.001 ; \mathrm{df}=2$; $F=64.2)$ and September $(P<0.001 ; \mathrm{df}=2,15$; $F=44 \cdot 6$ ).

Twelve species of cladocerans were found, with Bosmina longirostris the dominant organism (Table 3). Two density peaks occurred during June ( $8.56 \mathrm{ind} / \mathrm{l}$, site $1 ; 11.7 \mathrm{ind} / \mathrm{l}$, site 3 ) and July (15.7 ind/l, site 3 ). B. longirostris contributed to $100 \%$ and $94 \%$ of total density, respectively (Figure 3c). Significant differences in total density between sites were found in July $(P=0.0033$; $\mathrm{df}=2 ; F=8.6)$. Other cladocerans such as Alona costata, Alona rectangula and Chydorus sphaericus were detected consistently but at relatively low densities. Others including Ilyocryptus sordidus, Alonella nana and Alonella exigua, were detected intermittently.

High values for diversity $\left(\mathrm{H}^{\prime}>2\right)$ and equitability $\left(\mathrm{J}^{\prime}\right)$ indexes were registered in February (sites 1, 2 and 3), June (site 1), July (site 3), August (site 3), and September (sites 1, 2 and 3) (Figure 4a). The
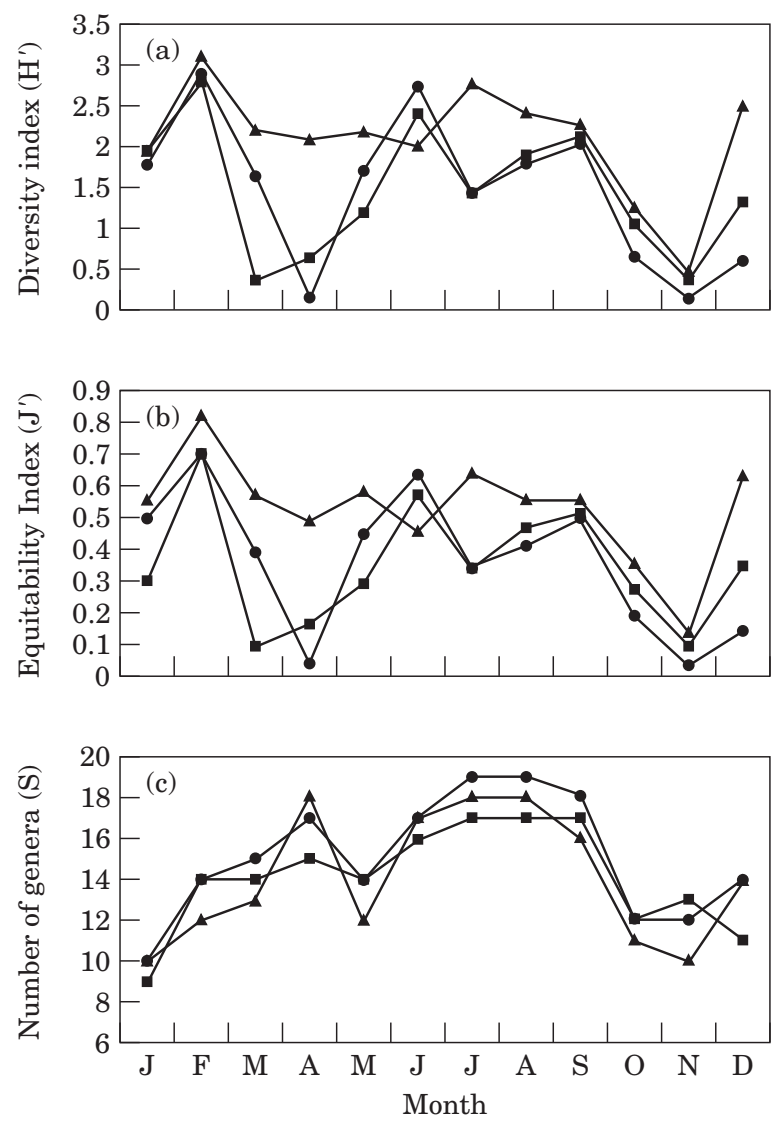

Figure 4. Values for (a) diversity, (b) equitability indexes

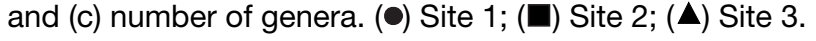

lowest $\mathrm{H}^{\prime}$ values were attained in March (site 2), April (sites 1 and 2), and November (sites 1, 2 and 3 ). $J^{\prime}$ values followed a similar trend (Figure $4 b$ ). The maximum number of genera was observed in July (site 1) and August (site 1). Site 3 showed a smaller variation in diversity $\left(\mathrm{H}^{\prime}\right)$ than sites 1 and 2. Higher temperatures tended to favour the development of a great number of taxa, but competition by nutrients should also be expected (Wetzel, 1993). A continuous decline in the number of genera was observed from September to December (Figure 4c).

To examine possible associations between zooplankton and other variables used to describe eutrophication, CA was performed. The three main axes explained $76.9 \%$ of the total variance. The first axis was defined by K. quadrata (Kqu), Anuraeopsis fissa (Afi), and April (Ap1, Ap2) in the negative part, and by $K$. cochlearis (Kch), H. mira (Hmir), July (Jl1, Jl2), August (Au1, Au2), October (Oc1, Oc2), November (N1, N2), and December (De1, De2, De3) in the positive part (Figure 5a). The first axis suggests the opposition between two main groups: the first characterised by the dominance 


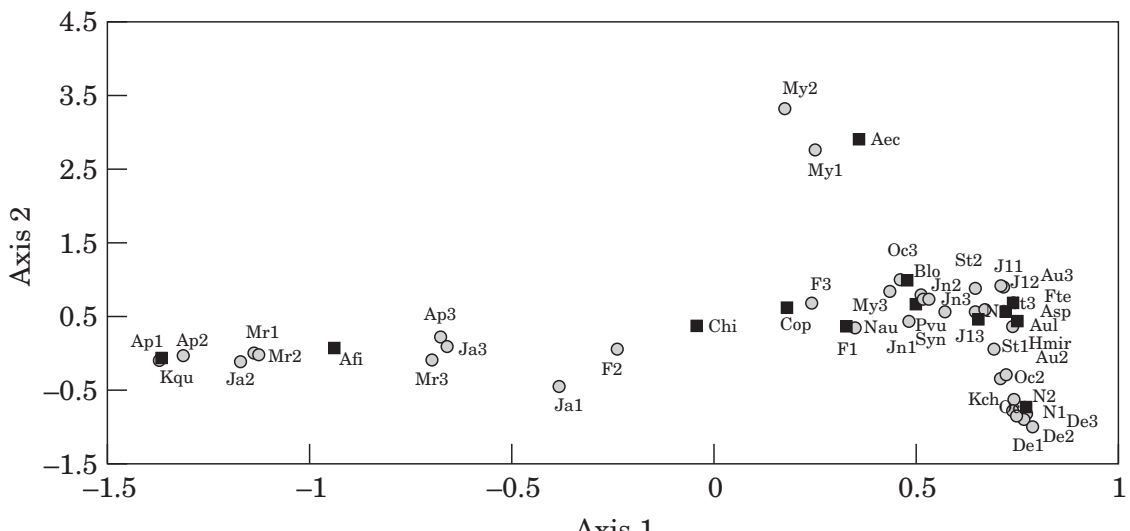

Axis 1
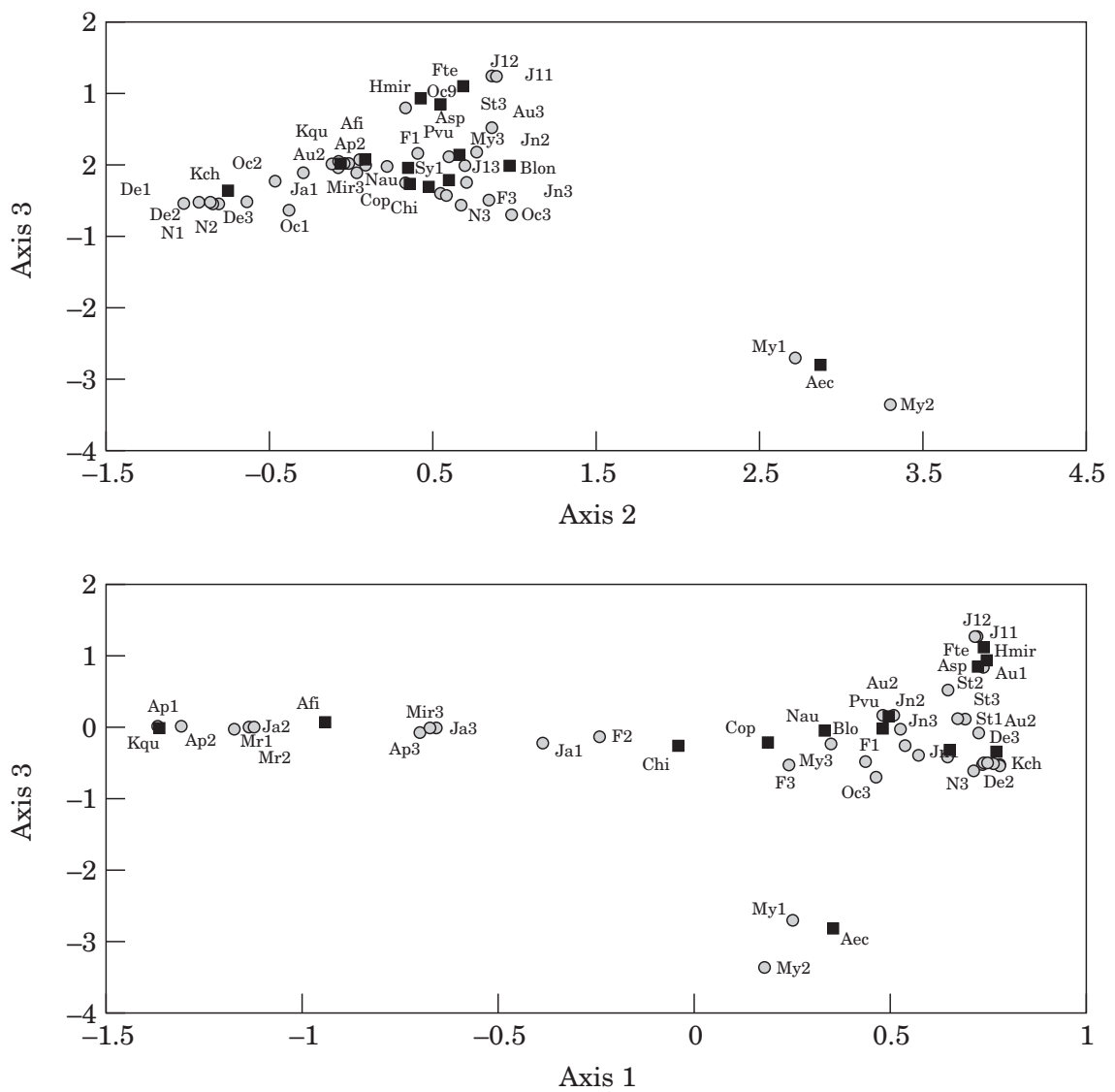

Figure 5. Correspondence analysis. Spatial representation of months and species for the three sampling sites: (a) plan 1X2, (b) plan 2X3 and (c) plan 1X3. Abbreviations: Ja-January; F - February; Mr - March; Ap - April; My - May; Jn - June; JI - July; Au - August; St - September; Oc - October; N - November; De - December; Fte - Filinia terminalis; Kqu -Keratella quadrata; Kch - Keratella cochlearis; Afi - Anuraeopsis fissa; Syn - Synchaeta spp.; Aec-Ascomorpha ecaudis; Pvu-Polyarthra vulgaris; Asp-Asplancna sp.; Hmir -Hexarthra mira; Blon-Bosmina longirostris; Chi-Chidoridae; $\mathrm{Nau}$-Nauplii; Cop -copepods). (ם) zooplanktonic taxa; $(\bigcirc)$ months/sampling sites.

of K. quadrata and A. fissa, and the second by the remaining species (Figure 5a). The polarity seemed to be determined by season with oxygen as the discriminating factor. K. quadrata and A. fissa have an analogous seasonal pattern, which may be confirmed by their consistent aggregation centred at the same temporal appearance. The lowest dissolved oxygen values were recorded in January and April. Dissolved oxygen used to be considered as an index of the water mass rather than an active factor in controlling rotifer abundance. However, it seems reasonable to argue that these organisms 
Table 4. Regression analysis of temperature, total suspended solids and chlorophyll-a on taxa densities [log $(\mathrm{DEN}+1)=\mathrm{a}+\operatorname{blog}(\mathrm{EF}+1)](\mathrm{DEN}-$ taxa densities; EF - environmental factor)

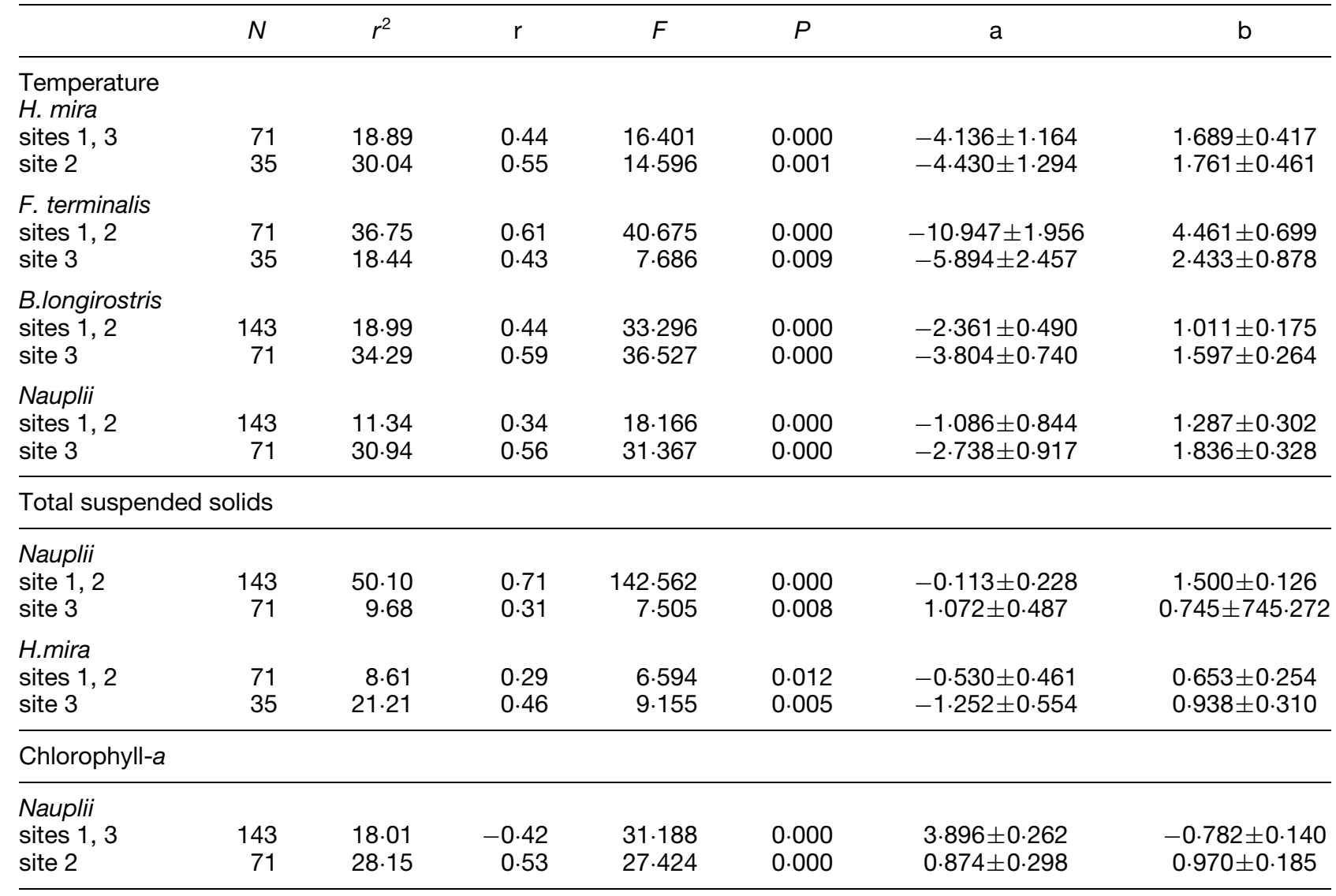

are influenced by dissolved oxygen, being tolerant to low concentrations.

The second axis was defined by $K$. cochlearis (Kch) and December (De1, De2, De3), in the negative part, and by Ascomorpha ecaudis (Aec) and May (My1, My2), in the positive part (Figure 5b). The third axis was defined by Ascomorpha ecaudis (Aec) and May (My1, My2), in the negative part, and by Filinia terminalis (Fte) and July (Jl1, Jl2) in the positive part (Figure 5c).

Statistically significant links $(P<0.05)$ between densities of the most abundant taxa and environmental factors were found only for $H$. mira, F. terminalis, Bosmina longirostris and Nauplii. Analysis of covariance (ANCOVA) also showed significant differences between slopes for the three sampling sites.

Temperature accounted for the total variability in the distribution of those taxa, namely: $19.0 \%$ $(P<0.001$, sites 1,3$)$ and $30.0 \%(P<0.001$, site 2) for Hexarthra mira; 36.8\% $(P<0.001$, sites 1 , $2)$ and $18.4(P=0.00897$, site 3$)$ for Filinia terminalis; $19.0 \%(P<0.001$, sites 1,2$)$ and $34.3 \%$ $(P<0.001$, site 3$)$ for Bosmina longirostris; $11.3 \%$
$(P<0.001$, sites 1,2$)$ and $30.9 \%(P<0.001$, site 3 ) for naupliar forms (Table 4). However, the distribution of naupliar forms was mainly associated with total suspended solids in site 1 and $2\left(r^{2}=50.1 \%, P<0.001\right)$. Chlorophyll- $a$ also for $18.01 \%(P<0.001$, sites 1,3$)$ and $28.2 \%(P<0.001$, site 2$)$ in the overall variation of naupliar densities (Table 4).

The abundance of Hexarthra mira was less strongly related to total suspended solids $\left(r^{2}=\right.$ $21.2 \%, P=0.0047$, site $3 ; r^{2}=8.6 \%, P=0.0124$, sites 1, 2) (Table 4).

\section{Discussion}

According to the classification proposed by Freedman (1989) for the trophic category of lakes and inland waters, the Lake could be considered to be hypereutrophic, based on its mean annual phosphorus concentration $(282 \mu \mathrm{g} / \mathrm{l})$ and minimum annual transparency $(0.5 \mathrm{~m})$. However, mean annual chlorophyll- $a$ concentration $\left(4.89 \mathrm{mg} / \mathrm{m}^{3}\right)$ places the Lake in the mesotrophic category. 
These observations suggested that, despite apparent nutrient availability, phytoplankton growth was inhibited in the Lake. This suggests a shift from a primary productivity based on phytoplankton to one based on macrophytes in natural ageing lakes (Björk, 1994a). Macrophytes could be the main factor responsible for phytoplankton inhibition in the Lake, through competition for light and nutrients (Moss, 1990; Wetzel, 1993; Jasser, 1995). Dissolved organic compounds resulting from organic matter mineralization may also create shade conditions and could also be a limiting factor to phytoplanktonic productivity (Carpenter et al., 1998). Chlorophyll- $a$ concentrations recorded in this study were lower than those registered by Gonçalves et al. (1996) in Mira lake, and by Vasconcelos (1990) (27 mg/m ${ }^{3}$ in June), in Braças lake, two eutrophic freshwater coastal lakes, located a few kilometres from Linhos. In the Lake, the dense coverage of macrophytes played an important role in water quality degradation. The large demand for oxygen to sustain the decomposition of this organic material accounted for the low values for dissolved oxygen obtained at depth. The decomposition of particulate organic matter is the main oxygen consuming process in deeper water (Wetzel, 1993).

Rotifers were by far the most dominant zooplanktonic group. A similar observation was recorded for some Sicilian mesotrophic water bodies (Flores and Barone, 1994). Their dominance in the Lake probably arose from their wide tolerance to variable dissolved oxygen concentrations (Galkovskaya, 1995) and from their capability to feed on bacteria and detritus (Ruttner-Kolisko, 1974; Habdija et al., 1993). The most abundant species-Keratella quadrata, K. cochlearis, Polyarthra vulgaris, Filinia terminalis and Hexarthra mira - are abundant in water bodies with a wide range of physico-chemical factors and are able to use bacteria and detritus in suspension as food resources (Bogdan and Gilbert, 1982; Zankai, 1989; Habdija et al., 1993; Ooms-Wilms, 1997). Macrophyte source detritus is likely the most important food source for rotifers in the Lake, since no correlations were found between these species and chlorophyll- $a$ concentrations. In natural conditions bacteria are difficult to assimilate; their food quality is poor as they lack some essential compounds for animal nutrition (Ooms-Wilms, 1997). The relative abundance of Filinia and Hexarthra, given their ability to feed on detritus, has been suggested as an indicator for mesotrophic water bodies (Mäemets, 1983). The representation of the copepod group almost exclusively by naupliar forms may be explained by a significant extended development times of these stages. This phenomenon is usually observed to be the result of a lack of good food resources (Hart, 1990).

The CA biplot of species and months showed three main groupings, each species grouped with its highest density month. However, species distribution follows a temperature gradient along axis 2 (Figure 5a), suggesting that temperature was the most important factor in determining seasonality. Regression analysis also indicated the influence of temperature on the abundance of Hexarthra mira, Filinia terminalis, Bosmina longirostris and naupliar forms, throughout the year.

$B$. longirostris (the most abundant cladoceran) displayed higher densities in June-July, after which it started to decrease. This could be explained by lower levels of dissolved oxygen and also by the high densities of Ceratium sp., which were recorded in zooplankton samples from August to September. This large edible species could not be eaten by small cladocerans, such as Bosmina (Auer et al., 1990).

The pattern of occurrence of $F$. terminalis was somewhat surprising. This species, considered as a cold stenothermic and hypolimnetic form (Ruttner-Kolisko, 1974), showed a density peak during June-July. This could be explained by the conjugation of high food availability with low oxygen concentrations.

Despite its small area, significant differences in total densities between sites for the three zooplanktonic groups were found in the Lake during density peaks. However, this could not be attributed to the environmental parameters measured since significant differences among sampling sites were not found. The efficiency with which different microcrustacean species can utilise non algal resources such as bacteria, protozoan (ciliates) and detritus may contribute to how the species selects the habitat (Smiley and Tessier, 1998). This was confirmed only for naupliar forms, whose distribution was related to that of chlorophyll- $a$ (Table 4) and total suspended solids (Table 4). A positive correlation was also found between $H$. mira densities and total suspended solids (Table 4) suggesting that detritus could be the main nutrient source for those organisms. Total densities of zooplanktonic groups also tended to increase from site 3 to site 1 , which may be due to passive drift within the small water area, as an increase in water level induces flow from site 3 to site 1 . Wind can also contribute to this spatial distribution, concentrating zooplankton in some areas. 
With regard to species diversity, values registered for the Lake were low, as expected. Reduced biodiversity is one of the features of eutrophication and subsequent terrestrialisation, as physicochemical conditions change (Brix and Schierup, 1989; Wetzel, 1993). The highest values, observed in February during this study, were not due to a high number of taxa. However, as shown by the high value of $\mathbf{J}^{\prime}$, diversity can increase without an increment in taxon number, if evenness increases (Hulbert, 1971). On the other hand, despite the high number of genera, diversity values registered in April were low, due to a density peak presented by Keratella quadrata.

The information provided by this first characterisation of environmental factors and zooplankton community structure in the Lake could be used for monitoring the effects of future restoration actions. Several restoration measures have been applied in shallow eutrophic lakes and the reports of their results can be used as theoretical foundations to derive and support restoration measures to be implemented in the Lake (Bettinetti et al., 1996; Moss et al., 1996; Søndergaard et al., 2000).

Perennial macrophytes such as Phragmites australis and Typha latifolia have morphological and physiological characteristics giving them ecological primacy in eutrophic systems. They can act as a pump pushing nutrients from the sediments (Odum, 1971; Björk, 1994b) which are incorporated and preserved in their structure during the growing season (Brix and Schierup, 1989; Björk, 1994b). Moreover, they have large internal air spaces for transporting oxygen to the roots and rhizomes, and can stimulate the decomposition of organic matter and the growth of nitrifying bacteria, creating oxidised conditions in a zone which is usually anoxic (Brix and Schierup, 1989). On this basis, annual cutting and removing of emergent macrophytes at the end of the growing season could be an important measure in reducing the internal load of nutrients in the Lake. The use of fire could be an alternative procedure, although the roots and rhizomes are not damaged by fire an increase in biomass in following years could be expected (Björk, 1994b). In addition, the following measures could be proposed:

(1) Cutting arboreal vegetation, mainly Eucalyptus globulus, near the banks, this will help reduce the exogenous input of organic matter due to falling leaves and increase water oxygenation by wind exposure and improve conditions for waterfowl flight.

(2) Increasing water area by controlling existing sluices and constructing an additional sluice to regulate water level.
(3) Periodic cleaning of ditches to reduce the input of organic matter due to surface runoff.

(4) Periodic clearing of undergrowth in the surrounding forest.

Additional measures, such as dredging of detritus covering the bottom of the Lake, should be planned if a system with sufficient longer term resilience capacity is to be obtained. The widespread anoxia in the sediment-water interface decreases the efficiency of phosphorus trapping in surface sediments (Quirós, 1990; Caraco, 1993); sediments could therefore be an important nutrient source to control. The removal of the detritus layer and underlying nutrient-rich sediments is probably the most effective method to improve water quality (Hosper and Jagtman, 1990; Nielsen, 1991; Björk, 1994c). Silva et al. (1997) described a partial dredging of lake-bottom sediments in Braças which improved water trophic conditions. Moss et al. (1996) reported a reduction in total phosphorus and chlorophyll concentrations, a colonization of macrophytes and an increase in Daphnia populations (abundance and body size) after sediment removal by suction dredging in a shallow eutrophic lake. However, the small water area of the Lake and its shallow depth may make mechanical intervention difficult. Phillips et al. (1994) have suggested that the high organic content and total phosphorus content of the remaining sediment after dredging may result in a large release of phosphorus in subsequent years. Other methods, such as the addition of phosphorus binding nutrients (aluminium sulphate, iron or calcium nitrate) (Nielsen, 1991; Romo and Bécares, 1994; Ripl, 1994; Welch and Schrieve, 1994; Søndergaard et al., 2000), coupled with the control of growing macrophytes, could be used as a possibly simple and cheaper alternative. However, though Søndergaard et al. (2000; unpublished data) have reported that the hypolimnetic addition of calcium nitrate was effective in the reduction of the internal release of phosphorus, permanent effects could not be obtained with a single dose. Romo and Bécares (1994) also confirmed that despite controlling nutrients in Madrid's urban lakes by the application of aluminium sulphate, additional measures were considered essential for long-term reduction in phytoplankton biomass. Additionally, aluminium sulphate and other chemicals may accumulate in the top sediment layer and may impair benthic communities (Bettinetti et al., 1996; Kleeberg et al., 2000).

Finally, hypolimnetic oxidation was tried as a restoration process in some Danish lakes, with the purpose of increasing the sediments' phosphorus binding capacity, thus enhancing the survival of 
aquatic organisms (Søndergaard et al., 2000). The results obtained suggested that this was not a costeffective process as it needs to be conducted for more than 12 years in order to produce permanent effects (Søndergaard et al., 2000).

In conclusion, zooplankton community structure proved to be a useful tool for characterizing the trophic state of a shallow water lake and identifying factors responsible for its water quality degradation. Further research is required to characterize the phytoplankton community structure and phytoplankton-zooplankton interactions. We believe that Linhos lake has reached the highest aquatic trophic state, characterised by enhanced phytoplanktonic primary production, and is now effectively a 'dead' ecosystem. We have proposed some restoration measures which we believe should be immediately implemented following the collection of further baseline information if the disappearance of the Lake is to be prevented, and its ecological quality restored.

\section{Acknowledgements}

The authors are very grateful to Pedro Raposo, M. J. Ferreira, P. C. Silva and other colleagues that helped with the fieldwork. This study was partially supported by Programa PRAXIS XXI in the form of a M.Sc. grant to R. Pereira.

\section{References}

Amoros, C. (1984). Introduction pratique à la systématique des organismes des eaux continentales françaises. Crustacés cladocères. Bulletin Mensuel de la Société Linnéenne de Lyon 4, 120-145.

APHA, AWWA and WPCF (1989). Standard Methods for Examination of Water and Wastewater. $17^{\text {th }}$ ed. Washington: American Public Health Association.

Auer, M. T., Storey, M. L., Effler, S. W., Auer, N. A. and Sze, P. (1990). Zooplankton impacts on chlorophyll and transparency in Onondaga Lake, New York, USA. Hydrobiologia 200/201, 603-617.

Bettinetti, A., Pypaert, P. and Sweerts, J.-P. (1996). Application of an integrated management approach to the restoration project of the Lagoon of Venice. Journal of Environmental Management 46, 207-227.

Björk, S. (1994a). The evolution of lakes and wetlands. In Restoration of Lake Ecosystem. A Holistic Approach (Martina Eiseltová, ed.), pp. 6-15. Slimbridge: IWRB.

Björk, S. (1994b). Macrophyte control. In Restoration of Lake Ecosystem. A Holistic Approach (Martina Eiseltová, ed.), pp. 89-96. Slimbridge: IWRB.

Björk, S. (1994c). Sediment removal. In Restoration of Lake Ecosystem. A Holistic Approach (Martina Eiseltová, ed.), pp. 82-88. Slimbridge: IWRB.

Bogdan, K. G. and Gilbert, J. J. (1982). Seasonal patterns of feeding by natural populations of Keratella,
Polyarthra and Bosmina: clearance rates, selectivities and contributions to community grazing. Limnology and Oceanography 27(5), 918-934.

Brix, H. and Schierup, H.-H. (1989). The use of aquatic macrophytes in water pollution control. Ambio 18, 100-107.

Brönmark, C. and Weisner, S. E. B. (1992). Indirect effects of fish community structure on submerged vegetation in shallow eutrophic lakes: an alternative mechanism. Hydrobiologia 234/244, 293-301.

Caraco, N. F. (1993). Disturbance of the phosphorus cycle: a case of indirect effects of human activity. Trends in Ecological Evolution 8(2), 51-54.

Carpenter, S. R., Cole, J. J., Kitchell, J. F. and Pace, M. L. (1998). Impact of dissolved organic carbon, phosphorus and grazing on phytoplankton biomass and production in experimental lakes. Limnology and Oceanography 43(1), 73-80.

Dussart, B. H. (1969). Les copépodes des eaux continentales d'Europe occidentale. Cyclopoïdes et biologie. Edition N. Boubée \& Cie.

Ejsmont-Karabin, J. (1995). Rotifer occurrence in relation to age, depth and trophic state of quarry lakes. Hydrobiologia 313/314, 21-28.

Flores, L. N. and Barone, R. (1994). Relationship between trophic state and plankton community structure in 21 Sicilian dam reservoirs. Hydrobiologia 275/276, 197-205.

Freedman, B. (1989). Environmental Ecology. The Impacts of Pollution and other Stresses on Ecosystem Structure and Function. San Diego, CA: Academic Press.

Galkovskaya, G. A. (1995). Oxygen consumption rate in rotifers. Hydrobiologia 313/314, 147-156.

Gliwicz, Z. M. (1990). Why do cladocerans fail to control algal blooms? Hydrobiologia 200/201, 83-97.

Gonçalves, F., Ribeiro, R., Vasconcelos, V. and Soares, A. M. V. M. (1996). Anthropogenic influences on seasonal changes of nutrients, physical and chemical factors in the three coastal freshwater shallow lakes (Portugal). Limnetica 12(2), 47-52.

Habdija, I., Primc-Habdija, B., Erben, R. and Belinić, I. (1993). Trophic role of rotifers in plankton of lake Kozjak (Plitvice Lakes). Hydrobiologia 257, 101-106.

Hart, R. C. (1990). Copepod post-embryonic durations: pattern, conformity and predictability. The realities of isochronal and equiproportional development and trends in the copepodid-naupliar duration ratio. Hydrobiologia 206, 175-206.

Hosper, S. H. and Jagtman, E. (1990). Biomanipulation additional to nutrient control for restoration of shallow lakes in the Netherlands. Hydrobiologia 200/201, 523-534.

Hulbert, S. H. (1971). The non concept of species diversity: a critique and alternative parameters. Ecology 52, 577-586.

Jasser, I. (1995). The influence of macrophytes on a phytoplankton community in experimental conditions. Hydrobiologia 306, 21-32.

Kleeberg, A., Nixdorf, B. and Mathes, J. (2000). Lake jabel restoration project: phosphorus status and possibilities and limitations of diversion of its nutrient-rich main inflow. Lakes \& Reservoirs: Research and Management 5, 23-33.

Klinge, M., Grimm, M. P. and Hosper, S. H. (1995). Eutrophication and ecological rehabilitation of dutch 
lakes: conceptual framework. Water Science and Technology 31(8), 207-218.

Legendre, L. and Legendre, P. (1979). Écologie Numérique. Le Traitement Multiple des Donnés Ecologiques. Tome 1, $1^{\text {st }}$ ed. Paris: Masson.

Mäemets, A. (1983). Rotifers as indicators of lake types in Estonia. Hydrobiologia 104, 357-361.

Magadza, C. H. D. (1994). Evaluation of eutrophication control in Lake Chivero, Zimbabwe, by multivariate analysis of zooplankton. Hydrobiologia 272, 277-292.

Marcin, T. C. (1995). Integrating social sciences into forest ecosystem management research. Journal of Forestry 29-33.

Matsubara, T. (1993). Rotifer community structure in the south basin of lake Biwa. Hydrobiologia 271, 1-10.

Michaud, M. T., Atchison, G. J., McIntosh, A. W., Mayes, R. A. and Nelson, D. W. (1979). Changes in phosphorus concentrations in a eutrophic lake as a result of macrophyte-kill following herbicide application. Hydrobiologia 66 (2), 105-111.

Moss, B. (1990). Engineering and biological approaches to the restoration from eutrophication of shallow lakes in which aquatic plant communities are important components. Hydrobiologia 200/201, 367-377.

Moss, B., Stansfield, J., Irvine, K., Perrows, M. and Phillips, G. (1996). Progressive restoration of a shallow lake: a 12-year experiment in isolation, sediment removal and biomanipulation. Journal of Applied Ecology 33, 71-86.

Nielsen, L. K. (1991). Water pollution. In Introduction to Environmental Management (P. E. Hansen and Jörgensen, eds), pp. 115-175. New York: Elsevier.

Odum, E. P. (1971). Fundamentos de Ecologia. $2^{\text {nd }}$ ed. Lisboa: Fundação Calouste Gulbenkian.

Ooms-Wilms, A. L. (1997). Are bacteria an important food source for rotifers in eutrophic lakes? Journal of Plankton Research 19, 1124-1141.

Pejler, B. (1983). Zooplanktonic indicators of trophy and their food. Hydrobiologia 101, 11-114.

Phillips, G., Jackson, R., Bennett, C. and Chilvers, A. (1994). The importance of sediment phosphorus release in the restoration of very shallow lakes (The Norfolk Broads, England) and implications for biomanipulation. Hydrobiologia 275/276, 445-456.

Pokórny, J. (1994). Development of aquatic macrophytes in shallow lakes and ponds. In Restoration of Lake Ecosystem. A Holistic Approach (Martina Eiseltová, ed.), pp. 36-43. Slimbridge: IWRB.

Power, M. E. (1992). Top-down and bottom-up forces in food webs: do plants have primacy? Ecology $\mathbf{7 3}(3)$, $733-746$.

Quirós, R. (1990). Factors related to variance of residuals in chlorophyll - total phosphorus regressions in lakes and reservoirs of Argentina. Hydrobiologia 200/201, 343-355.
Ripl, W. (1994). Sediment treatment. In Restoration of Lake Ecosystem. A Holistic Approach (Martina Eiseltová, ed.), pp. 75-81. Slimbridge: IWRB.

Rohlf, F. J. (1992). Numerical Taxonomy and Multivariate Analysis System. New York: Exeter Software.

Romo, S. and Bécares, E. (1994). Water management of two shallow urban eutrophic lakes. Water Science and Technology 30, 299-302.

Ruttner-Kolisko, A. (1974). Plankton rotifers. Biology and taxonomy. Suppl. Die Binnengewässer, pp. 1-145. Stuttgart: Schweizerbart'sch Verlagsbuchhandlung.

Scourfield, D. J. and Harding, J. P. (1966). A key to the British freshwater cladocera. Freshwater Biological Association Scientific Publication 5.

Seip, K. L. (1994). Phosphorus and nitrogen limitation of algal biomass across trophic gradients. Aquatic Sciences 56(1), 16-27.

Silva, P. C. L. D., Gonçalves, F., Ribeiro, R. and Soares, A. M. V. M. (1997). First evaluation of the restoration of Braças lagoon (Figueira da FozPortugal). Archives Hydrobiology 141, 109-125.

Smiley, E. A. and Tessier, A. J. (1998). Environmental gradients and the horizontal distribution of microcrustaceans in lakes. Freshwater Biology 39, 397-409.

Søndergaard, M., Jeppesen, E., Jensen, J. P. and Lauridsen, T. (2000). Lake restoration in Denmark. Lakes \& Reservoirs: Research and Management 5, 151-159.

Uku, J. N. and Mavuti, K. M. (1994). Comparative limnology, species diversity and biomass relationship of zooplankton and phytoplankton in five freshwater lakes in Kenya. Hydrobiologia 272, 251-258.

Vasconcelos, V. (1990). Ecotoxicologia de Cianobactérias: Impacte Dos seus 'Blooms' nas Comunidades Animais e Sua Aplicação na Gestão da Qualidade da água. Provas de aptidão científica e pedagógica. Porto: Faculdade de Ciências do Porto.

Vasconcelos, V. (1994). Toxic cyanobacteria (blue-green algae) in Portuguese fresh waters. Archives Hydrobiology 130, 439-451.

Washington, H. G. (1984). Diversity, biotic and similarity indices. A review with special relevance to aquatic ecosystems. Water Research 1, 164-172.

Welch, E. B. and Schrieve, G. D. (1994). Alum treatment effectiveness and longevity in shallow lakes. Hydrobiologia 275/276, 423-431.

Wetzel, R. G. (1993). Limnologia. $2^{\text {nd }}$ ed. Lisboa: Fundação Calouste Gulbenkian.

Zankai, N. P. (1989). Horizontal distribution of rotifer plankton along a trophic gradient in lake Balaton: changes of community structure and abundance during the past 20 years. Archives Hydrobiologia 115, 111-123.

Zar, J. H. (1996). Biostatistical Analysis, $3^{\text {rd }}$ ed. Englewood Cliffs, NJ: Prentice-Hall. 